Revista Brasil. Bot., V.31, n.4, p.633-644, out.-dez. 2008

\title{
Padrões de distribuição geográfica das espécies de Schefflera J. R. Forst. \& G. Forst. (Araliaceae) do Brasil extra-amazônico ${ }^{1}$
}

\author{
PEDRO FIASCHI ${ }^{2,4}$ e JOSÉ RUBENS PIRANI ${ }^{3}$
}

(recebido: 26 de outubro de 2006; aceito: 1 de setembro de 2008)

\begin{abstract}
Patterns of geographic distribution of the extra-Amazonian Brazilian species of Schefflera J. R. Forst. \& G. Forst. (Araliaceae). An analysis of the geographic distribution of the 26 species of Schefflera from extra-Amazonian Brazil was prepared based on maps depicting the known occurrences of the taxa. These include: S. angustissima (Marchal) Frodin, S. aurata Fiaschi, S. botumirimensis Fiaschi \& Pirani, S. burchellii (Seem.) Frodin \& Fiaschi, S. calva (Cham.) Frodin \& Fiaschi, S. capixaba Fiaschi, S. cephalantha (Harms) Frodin, S. cordata (Taub.) Frodin \& Fiaschi, S. distractiflora (Harms) Frodin, S. fruticosa Fiaschi \& Pirani, S. gardneri (Seem.) Frodin \& Fiaschi, S. glaziovii (Taub.) Frodin \& Fiaschi, $S$. grandigemma Fiaschi, S. kollmannii Fiaschi, S. longipetiolata (Pohl ex DC.) Frodin \& Fiaschi, S. lucumoides (Decne. \& Planch. ex Marchal) Frodin \& Fiaschi, S. macrocarpa (Cham. \& Schltdl.) Frodin, S. malmei (Harms) Frodin, S. morototoni (Aubl.) Maguire, Steyermark \& Frodin, S. racemifera Fiaschi \& Frodin, S. ruschiana Fiaschi \& Pirani, S. selloi (Marchal) Frodin \& Fiaschi, S. succinea Frodin \& Fiaschi, S. villosissima Fiaschi \& Pirani, S. vinosa (Cham. \& Schltdl.) Frodin \& Fiaschi e $S$. aff. varisiana Frodin. Two centers of endemism associated with high-altitude areas were recognized: Espinhaço Range in Minas Gerais state and montane forests of Espírito Santo state. The geographic patterns found are discussed with regard to other groups of angiosperms, as well as to phytogeographic studies of the main extra-Amazonian Brazilian phytocoria. Some hypothesis concerning probable phylogenetic relationships among some taxa are analysed in the light of the observed distribution patterns.
\end{abstract}

Key words - Brazil, Didymopanax, endemism, geographic distribution, Schefflera

RESUMO - (Padrões de distribuição geográfica das espécies de Schefflera J. R. Forst. \& G. Forst. (Araliaceae) do Brasil extra-amazônico). Uma análise da distribuição geográfica de Schefflera no Brasil extra-amazônico foi realizada com base em mapas atualizados plotando as ocorrências conhecidas das 26 espécies do gênero encontradas nessa grande área: $S$. angustissima (Marchal) Frodin, S. aurata Fiaschi, S. botumirimensis Fiaschi \& Pirani, S. burchellii (Seem.) Frodin \& Fiaschi, S. calva (Cham.) Frodin \& Fiaschi, S. capixaba Fiaschi, S. cephalantha (Harms) Frodin, S. cordata (Taub.) Frodin \& Fiaschi, S. distractiflora (Harms) Frodin, S. fruticosa Fiaschi \& Pirani, S. gardneri (Seem.) Frodin \& Fiaschi, S. glaziovii (Taub.) Frodin \& Fiaschi, S. grandigemma Fiaschi, S. kollmannii Fiaschi, S. longipetiolata (Pohl ex DC.) Frodin \& Fiaschi, S. lucumoides (Decne. \& Planch. ex Marchal) Frodin \& Fiaschi, S. macrocarpa (Cham. \& Schltdl.) Frodin, S. malmei (Harms) Frodin, $S$. morototoni (Aubl.) Maguire, Steyermark \& Frodin, S. racemifera Fiaschi \& Frodin, S. ruschiana Fiaschi \& Pirani, S. selloi (Marchal) Frodin \& Fiaschi, S. succinea Frodin \& Fiaschi, S. villosissima Fiaschi \& Pirani, S. vinosa (Cham. \& Schltdl.) Frodin \& Fiaschi e $S$. aff. varisiana Frodin. Dois centros de endemismo associados com áreas de altitude elevada foram reconhecidos: Cadeia do Espinhaço em Minas Gerais e florestas montanas do Estado do Espírito Santo. Os padrões de distribuição geográfica ilustrados são discutidos com base em dados obtidos para outros grupos de angiospermas e em estudos fitogeográficos das principais fitocórias do Brasil extra-amazônico. São apresentadas também hipóteses acerca de prováveis relações filogenéticas entre alguns táxons, visando à busca de possíveis correlações entre estas e a biogeografia do grupo.

Palavras-chave - Brasil, Didymopanax, distribuição geográfica, endemismo, Schefflera

\section{Introdução}

O gênero Schefflera conta com 600-900 espécies, sendo muitas ainda inéditas (Frodin \& Govaerts 2003,

1. Parte da dissertação de mestrado do primeiro autor, programa de Pós graduação em Botânica do Instituto de Biociências da USP.

2. Department of Biology, Virginia Commonwealth University, 1000 West Cary Street, P.O. Box 842012, Richmond, VA, U.S.A.

3. Universidade de São Paulo, Instituto de Biociências, Departamento de Botânica, Rua do Matão, Travessa 14, 321, 05508-900 São Paulo, SP, Brasil.

4._Autor para correspondência: pedrofiaschi@hotmail.com
Frodin 2004). Na delimitação atual, Schefflera é grupo polifilético, resultando do agrupamento de cinco clados independentes, um dos quais abrangendo as espécies neotropicais (Plunkett et al. 2004, 2005, Wen et al. 2001).

Os principais centros de diversidade específica do gênero encontram-se em regiões tropicais do Sudeste da Ásia e Indonésia, Ilhas do Sudoeste do Oceano Pacífico, e Américas Central e do Sul (Frodin 1995). Na Região Neotropical, onde ocorrem 250-300 espécies (Frodin 2004), seus centros de diversidade estão nos Andes e América Central (ca. 180 spp.), e no Planalto das Guianas e áreas vizinhas ( $c a$. 60 ou mais spp.). No Brasil o gênero encontra- 
se mais bem representado na Costa Atlântica e nos Planaltos Central (principalmente na Cadeia do Espinhaço) e das Guianas (Fiaschi \& Pirani 2005a, 2005b, Frodin 1995).

As espécies neotropicais de Schefflera foram organizadas por Frodin (1995) em sete grupos infragenéricos informais, dos quais quatro ("Cheilodromi", "Crepinella", "Didymopanax" e "Sciodaphyllum") possuem espécies nativas no Brasil. Excetuando-se "Didymopanax", cuja maioria das espécies ocorre no Brasil, os demais grupos são especialmente diversos em áreas peri-Amazônicas do norte da América do Sul (Granville 1992), tais como o Planalto das Guianas ("Cheilodromi" e "Crepinella") e as Florestas Andinas ("Sciodaphyllum"). Este último, cuja delimitação atual é bastante heterogênea, possui distribuição ampla desde a América Central até a Bolívia e o Planalto da Guianas, além de ocorrer em áreas do Velho Mundo (Frodin 1995).

A maioria das $c a .50$ espécies brasileiras de Schefflera pertence ao grupo "Didymopanax", que, embora tenha sido considerado durante muito tempo como um gênero à parte, consta atualmente como sinônimo do primeiro. Frodin (1995) reconheceu dois grupos dentro de "Didymopanax": "Confusae", incluindo espécies amazônicas com ovário 5-6-locular [p.e. Schefflera confusa (Marchal) Harms, S. megacarpa A.H. Gentry], e "Didymopanax", incluindo espécies com com ovário geralmente 2(-3)-locular e diversidade centrada no Leste do Brasil (e.g. Schefflera angustissima, S. macrocarpa e $S$. vinosa). É a este último grupo que pertence a maioria das espécies de Schefflera do Brasil extra-amazônico, objeto do presente estudo, que visou mapear sua distribuição geográfica e discutir os padrões obtidos com dados obtidos sobre outros táxons e estudos fitogeográficos regionais. O conhecimento da distribuição geográfica de espécies de "Didymopanax" constitui o primeiro passo para a compreensão dos processos responsáveis pela diversificação do grupo em áreas do leste da América do Sul, o que será abordado futuramente. A síntese biogeográfica aqui apresentada deriva de estudos taxonômicos conduzidos pelos autores (Fiaschi 2004, Fiaschi \& Pirani 2005a, 2005b, 2007, Fiaschi \& Frodin 2006).

\section{Material e métodos}

Os mapas de distribuição geográfica das 26 espécies de Schefflera listadas na tabela 1 foram modificados a partir do Mapa Base número 1 da Flora Neotropica (Dept. of Systematic Botany, State University of Utrecht) e do Mapa da Cadeia do Espinhaço em Minas Gerais de Rapini (2000). Foram plotadas as localidades de ocorrência comprovada pelo exame de materiais depositados nos seguintes herbários, de acordo com Holmgren et al. (1990): ALCB, BHCB, BM, BR, C, CEPEC, CESJ, CVRD, ESA, GFJP, HRCB, HUEFS, INPA, K, M, MBM, MBML, MG, MO, NY, P, R, RB, S, SP, SPF, UB, UEC, US, VIES, W e WU.

Constam nos mapas apenas os pontos correspondentes aos materiais analisados. Informações disponíveis em referências bibliográficas não foram consideradas devido a recorrentes imprecisões na identificação do material.

A definição de domínios morfoclimáticos e vegetacionais seguida é a de Ab'Saber (1967), discutida e atualizada por Joly et al. (1999).

\section{Resultados e discussão}

As 26 espécies de Schefflera que ocorrem no Brasil extra-amazônico (tabela 1) podem ser agrupadas em dois blocos correspondentes a dois dos domínios fitogeográficos que mais contribuem na formação das paisagens naturais dessa Região: o Cerrado e a Mata Atlântica (incluindo as fitocórias "Complexo das Florestas Atlânticas" e "Sul do Brasil"; Daly \& Mitchell 2000). As únicas espécies que não se encaixam de forma satisfatória nesses dois grupos são Schefflera morototoni, cuja distribuição geográfica é ampla na região Neotropical (figura 1a), e $S$. calva e $S$. aff. varisiana, que embora sejam encontradas na Mata Atlântica, penetram no domínio do Cerrado por meio das matas ciliares (figura 1b) e florestas montanas (figura 1e). Ainda restam dúvidas se os materiais aqui referidos como $S$. aff. varisiana correspondem à mesma espécie encontrada no Planalto das Guianas (S. varisiana Frodin) ou se sua distribuição é restrita a áreas da Mata Atlantica e Cadeia do Espinhaço.

Tanto no Cerrado como na Mata Atlântica o gênero Schefflera encontra-se melhor representado em áreas situadas em altitudes elevadas, sendo bastante freqüentes os casos de endemismo em campos rupestres e florestas montanas. No domínio do Cerrado merece destaque a porção mineira da Cadeia do Espinhaço, onde Schefflera parece ter sofrido uma diversificação significativa, assim como já foi sugerido em diversos gêneros, como Leiothrix (Eriocaulaceae), Pseudotrimezia (Iridaceae), Senna (Leguminosae), Eriope (Lamiaceae) e Barbacenia (Velloziaceae) (Giulietti \& Pirani 1988, Harley 1988, MelloSilva 1995). Na Mata Atlântica uma maior diversificação de Schefflera teria supostamente ocorrido na porção setentrional da Serra do Mar, no Rio de Janeiro, e na região serrana do Espírito Santo, principalmente em áreas ao sul do Rio Doce.

Cerrado - espécies de formações campestres e savânicas. Estudos recentes visando uma melhor compreensão da 
Tabela 1. Espécies de Schefflera ocorrentes no Brasil extra-amazônico, domínios fitogeográficos em que aparecem $(\mathrm{CE}=$ cerrados; MA = Mata Atlântica; AM = Amazônia) e estados onde podem ser encontradas.

Table 1. Extra-Amazonian Brazilian species of Schefflera, their phytogeographic domains $(\mathrm{CE}=\mathrm{Cerrado}$; MA = Atlantic rainforests; $\mathrm{AM}=\mathrm{Amazon}$ ), and the states where they can be found.

\begin{tabular}{|c|c|c|}
\hline Espécies & Domínios & Estados \\
\hline Schefflera angustissima (Marchal) Frodin & MA & BA, MG, ES, RJ, SP, PR, SC \\
\hline S. aurata Fiaschi & MA & $\mathrm{BA}$ \\
\hline S. botumirimensis Fiaschi \& Pirani & $\mathrm{CE}$ & MG \\
\hline S. burchellii (Seem.) Frodin \& Fiaschi & $\mathrm{CE}$ & MA, BA, GO, TO, MT \\
\hline S. calva (Cham.) Frodin \& Fiaschi & MA, CE & GO, MG, ES, RJ, SP, PR \\
\hline S. capixaba Fiaschi & MA & ES \\
\hline S. cephalantha (Harms) Frodin & $\mathrm{CE}$ & MT \\
\hline S. cordata (Taub.) Frodin \& Fiaschi & $\mathrm{CE}$ & MG \\
\hline S. distractiflora (Harms) Frodin & $\mathrm{CE}$ & AM, PA, RO, MT, GO \\
\hline S. fruticosa Fiaschi \& Pirani & $\mathrm{CE}$ & MG \\
\hline S. gardneri (Seem.) Frodin \& Fiaschi & $\mathrm{CE}$ & MG \\
\hline S. glaziovii (Taub.) Frodin \& Fiaschi & $\mathrm{CE}$ & MG \\
\hline S. grandigemma Fiaschi & MA & ES \\
\hline S. kollmannii Fiaschi & MA & ES \\
\hline S. longipetiolata (Pohl ex DC.) Frodin \& Fiaschi & MA & MG, ES, RJ \\
\hline $\begin{array}{l}\text { S. lucumoides (Decne. \& Planch. ex Marchal) } \\
\text { Frodin \& Fiaschi }\end{array}$ & $\mathrm{CE}$ & MG \\
\hline S. macrocarpa (Cham. \& Schltdl.) Frodin & $\mathrm{CE}$ & $\mathrm{BA}, \mathrm{GO}, \mathrm{MG}, \mathrm{SP}$ \\
\hline S. malmei (Harms) Frodin & $\mathrm{CE}$ & MT, MS, GO, SP \\
\hline $\begin{array}{l}\text { S. morototoni (Aubl.) Maguire, Steyermark \& } \\
\text { Frodin }\end{array}$ & MA, CE, AM & $\begin{array}{l}\text { AM, AP, RR, RO, PA, MT, MS, TO, GO, } \\
\text { MA, PI, CE, RN, PB, PE, AL, SE, BA, ES, } \\
\text { MG, RJ, SP, PR }\end{array}$ \\
\hline S. racemifera Fiaschi \& Frodin & MA & ES \\
\hline S. ruschiana Fiaschi \& Pirani & MA & ES \\
\hline S. selloi (Marchal) Frodin \& Fiaschi & MA & BA, ES \\
\hline S. succinea Frodin \& Fiaschi & MA & RJ \\
\hline S. villosissima Fiaschi \& Pirani & $\mathrm{CE}$ & MG \\
\hline S. vinosa (Cham. \& Schltdl.) Frodin \& Fiaschi & $\mathrm{CE}$ & BA, GO, MS, MG, SP, PR \\
\hline S. aff. varisiana Frodin. & $\mathrm{MA}, \mathrm{CE}$ & $\mathrm{BA}, \mathrm{MG}, \mathrm{ES}$ \\
\hline
\end{tabular}

flora do Cerrado têm revelado uma elevada diversidade de angiospermas (Castro et al. 1999) e uma heterogeneidade florística significativa, o que tem fornecido bases sólidas para o reconhecimento de agrupamentos florísticos distintos nesse domínio (Ratter et al. 1996, Oliveira-Filho \& Ratter 2002), e sua inclusão como área prioritária para conservação da biodiversidade (hotspot, Myers et al. 2000). Assim, estudos de táxons com elevada diversidade nos cerrados servem como indicadores das afinidades florísticas entre áreas pertencentes a esse domínio, fornecendo subsídios para a conservação de locais que abrigam elevados níveis de endemismo ou aquelas ainda não protegidas nas unidades de conservação existentes (Simon \& Proença 2000).

Tendo o Planalto Central como um de seus centros de diversidade no Brasil (Frodin 1995), Schefflera conta com 13 espécies em formações campestres e savânicas do Cerrado. Destas, sete são endêmicas de áreas com 


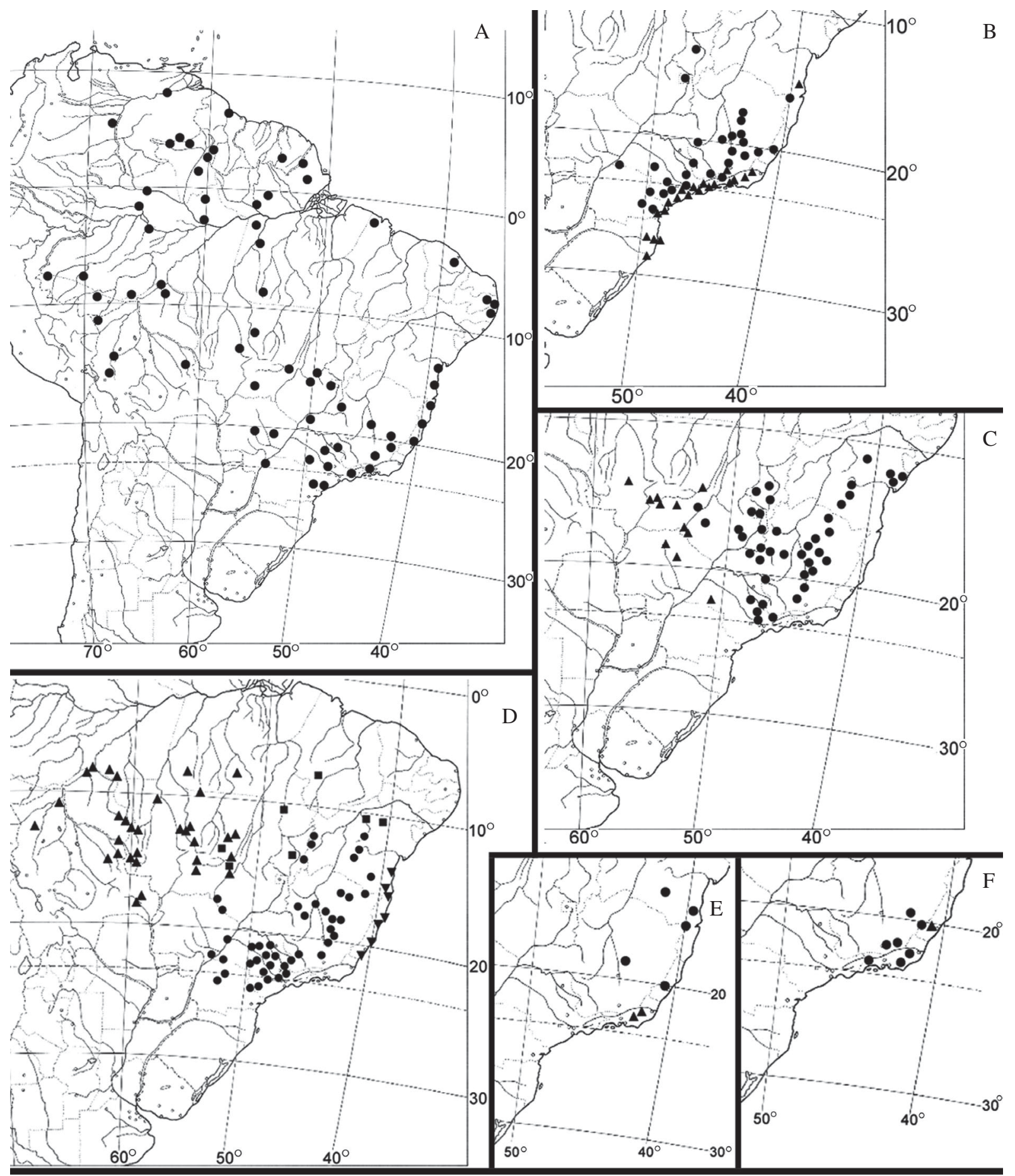

Figura 1. A. Distribuição geográfica de $S$. morototoni ( • ). B. Distribuição geográfica de $S$. angustissima ( $\mathbf{\Delta})$ e $S$. calva

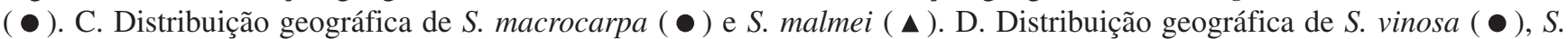

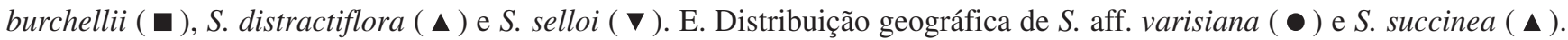
F. Distribuição geográfica de $S$. longipetiolata $(\bullet)$ e $S$. racemifera ( $\boldsymbol{\Delta}$ ).

Figure 1. A. Geographic distribution of S. morototoni (•). B. Geographic distribution of S. angustissima ( $\mathbf{\Delta})$ and S. calva

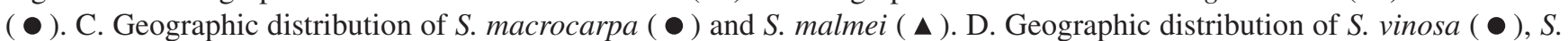

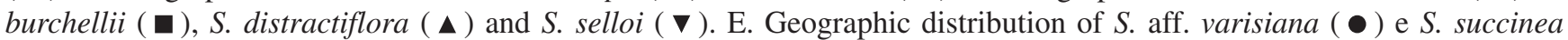
( $\Delta$ ). F. Geographic distribution of S. longipetiolata $(\bullet)$ and S. racemifera ( $\Delta$ ). 
altitude acima de $1000 \mathrm{~m}$ na Cadeia do Espinhaço em Minas Gerais, cuja área corresponde a menos de $5 \%$ da área total ocupada pelo bioma ( $c a .2$ milhões de $\mathrm{km}^{2}$ ). As seis demais espécies são encontradas em cerrados de terrenos baixos, situados entre 300 e $600 \mathrm{~m}$ alt., cuja área abrange $c a$. $50 \%$ da área total ocupada pelo Cerrado (Dias 1996 apud Simon \& Proença 2000).

i) Cerrados de terrenos baixos. Das espécies ocorrentes na região Sudeste que habitam cerrados de terrenos baixos, Schefflera macrocarpa é a mais amplamente distribuída, com ocorrência concentrada em porções orientais do domínio, geralmente entre os paralelos $40^{\circ}$ e $50^{\circ} \mathrm{W}$ (figura 1c). Já Schefflera malmei, aqui tratada como um par vicariante de $S$. macrocarpa, distribui-se em áreas mais ocidentais, tendo como limite de distribuição oriental o oeste do Estado de São Paulo, próximo ao paralelo $50^{\circ} \mathrm{W}$ (figura 1c). Apesar das áreas de distribuição de ambas não serem completamente disjuntas, é possível reconhecê-las mesmo quando faltam dados precisos sobre a localidade, razão que nos levou a não seguir a sinonimização proposta por Moura (1983) (Fiaschi \& Pirani 2007).

Schefflera vinosa também possui distribuição geográfica ampla em cerrados do sudeste do Brasil, sendo mais freqüentemente encontrada em áreas periféricas do limite meridional do Cerrado (em São Paulo e norte do Paraná), e nos cerrados de altitude e campos rupestres da Cadeia do Espinhaço em Minas Gerais e Bahia (figura 1d). Essa extensão setentrional ao longo das serras do Espinhaço está provavelmente associada ao efeito altitudinal compensatório em latitudes inferiores, uma vez que os registros de ocorrência de $S$. vinosa ao longo da Cadeia do Espinhaço são correlacionados com as áreas de altitudes elevadas. Fiaschi \& Pirani (2007) trataram S. vinosa como uma ocloespécie (White 1962, Cronq 1998), no entanto há populações cuja variabilidade fenotípica e distribuição geográfica parecem consistentes para que sejam reconhecidas taxonomicamente, o que será avaliado em estudos futuros.

As demais três espécies de Schefflera encontradas em formações savânicas do Cerrado não ocorrem na região Sudeste do Brasil. Schefflera burchellii distribui-se ao norte de Brasília, da Chapada dos Veadeiros (Goiás) até o sul do Maranhão, com extensão oeste até a porção oriental do Mato Grosso, e leste na região de Campo Formoso (Bahia), em serras pertencentes à Cadeia do Espinhaço (figura 1d). Esta espécie assemelha-se bastante a $S$. vinosa, da qual difere pelos folíolos elípticos (ovados) e peciolulados, e pelos ramos espessados e corticosos. Em áreas ainda mais ocidentais, são presentes no Cerrado
Schefflera distractiflora e $S$. cephalantha. A primeira é frequiente no Mato Grosso, com extensões a oeste nos estados de Rondônia e na Bolívia, e limite setentrional no sul dos estados do Amazonas e Pará (figura 1d). A segunda é conhecida apenas pelo material-tipo (Malme 2073), coletado em área onde atualmente encontra-se o Parque Nacional da Chapada dos Guimarães (Mato Grosso) (figura 2c).

Tanto $S$. burchellii quanto $S$. distractiflora possuem áreas de distribuição geográfica mais ou menos concordantes com os grupos geográficos propostos como constituintes do domínio do Cerrado (Ratter et al. 1996), a primeira encaixando-se no grupo norte e a segunda no grupo centro-ocidental.

ii) Endemismo - Cadeia do Espinhaço. Como dito anteriormente, sete das 13 espécies que habitam formações abertas do domínio dos cerrados possuem distribuição geográfica restrita à Cadeia do Espinhaço em Minas Gerais. Dessas, nenhuma se distribui ao longo de toda a extensão da Serra do Espinhaço (nome usado para designar o setor mineiro da formação, sendo a Chapada Diamantina o seu setor baiano), uma vez que são constituídas por populações com áreas de distribuição restrita, raramente sendo encontradas em formações montanhosas descontínuas.

Casos de distribuição geográfica descontínua em serras da Cadeia do Espinhaço são recorrentes em plantas arbustivas e herbáceas dos campos rupestres (Giulietti \& Pirani 1988), padrão aqui ilustrado por Schefflera gardneri. São encontradas populações desta espécie desde o Planalto de Diamantina até a Serra de Itacambira, entre os Municípios de Itacambira e Juramento (nordeste de Minas Gerais), e na Serra do Cabral, deslocada a noroeste do Planalto de Diamantina (figura 2a). Por outro lado, duas espécies a ela supostamente relacionadas (Fiaschi \& Pirani 2007), Schefflera botumirimensis e S. glaziovii, constituem casos notáveis de endemismo restrito a apenas uma serra, padrão também reconhecido por Giulietti \& Pirani (1988). A primeira é conhecida apenas da Serra da Canastra, em Botumirim, na porção norte da Serra do Espinhaço, enquanto a segunda é conhecida apenas de alguns municípios da porção norte da Serra do Cipó (figura 2a).

Esse parece ser o caso também de Schefflera villosissima e $S$. cordata, ambas supostamente relacionadas a S. macrocarpa e endêmicas do Planalto de Diamantina. Schefflera villosissima possui distribuição geográfica restrita à porção oriental do Planalto de Diamantina e à Serra do Ambrósio, posicionada a leste, mas em latitude semelhante à do Planalto (figura $2 b$ ), locais onde apesar 
de ocorrer em simpatria com $S$. macrocarpa, mantém-se distinta desta pelo denso indumento e unidades terminais das inflorescências capituladas. Schefflera cordata, por

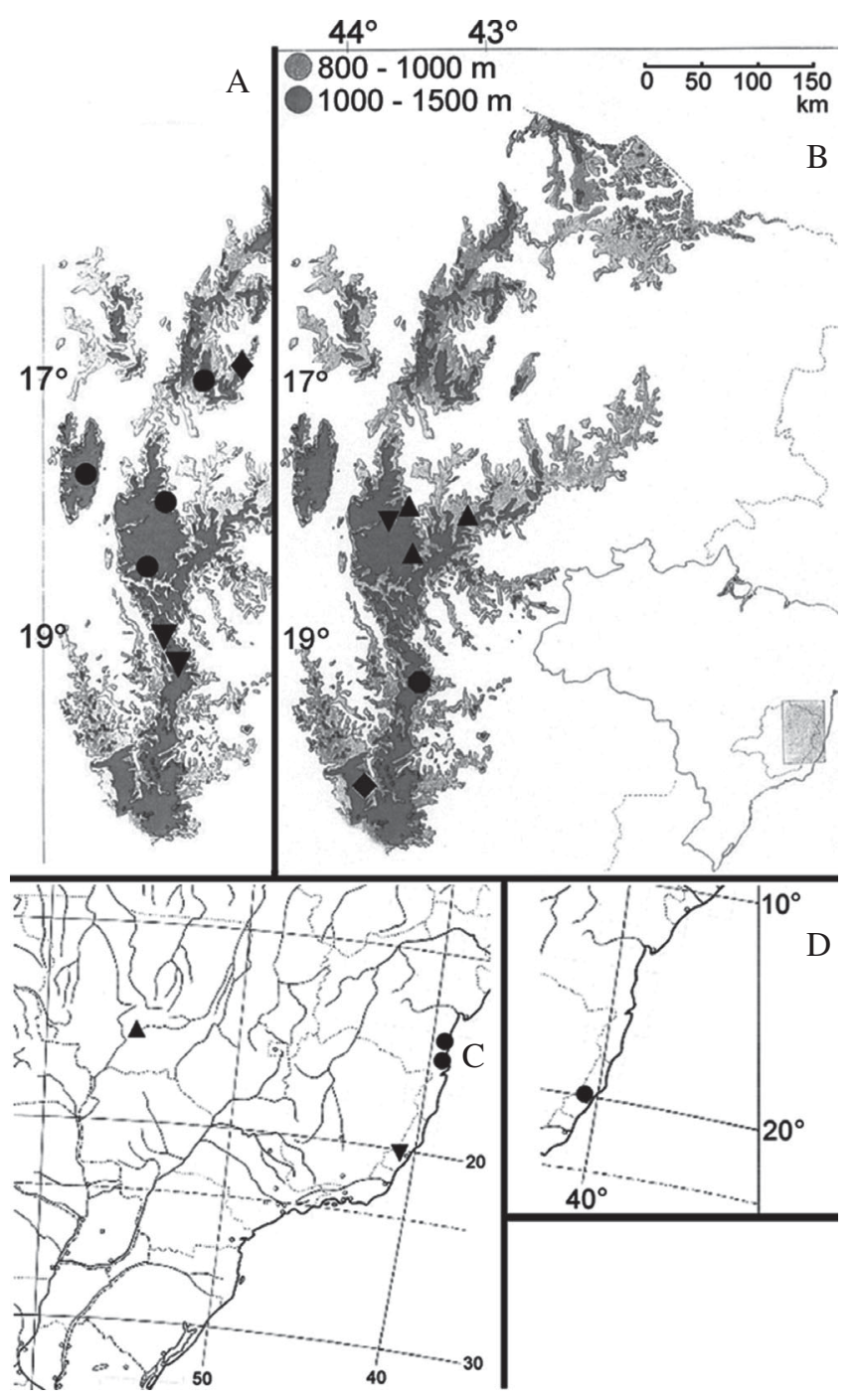

Figura 2. A. Distribuição geográfica de $S$. gardneri (• ), $S$. glaziovii $(\boldsymbol{\nabla})$ e $S$. botumirimensis $(\diamond)$. B. Distribuição geográfica de $S$. villosissima ( $\mathbf{\Delta}$ ), S. cordata ( $\mathbf{\nabla})$, S. fruticosa (•) e $S$. lucumoides ( $\bullet$ ). C. Distribuição geográfica de $S$. aurata ( ), S. capixaba ( $\boldsymbol{\nabla})$ e S. cephalantha $(\boldsymbol{\Delta})$. D. Distribuição geográfica de $S$. grandigemma, S. kollmannii e S. ruschiana (•).

Figure 2. A. Geographic distribution of S. gardneri (•), $S$. glaziovii $(\boldsymbol{\nabla})$ and $S$. botumirimensis $(\bullet)$. B. Geographic distribution of S. villosissima ( $\mathbf{\Delta})$, S. cordata $(\boldsymbol{\nabla}), S$. fruticosa (•) and S. lucumoides $(\bullet)$ ). C. Geographic

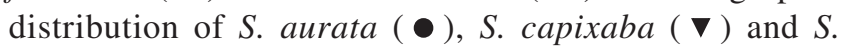
cephalantha ( $\boldsymbol{\Delta})$. D. Geographic distribution of $S$. grandigemma, S. kollmannii and S. ruschiana (•). sua vez, ocorre apenas no Planalto de Diamantina, nas proximidades de Mendanha e São João da Chapada (figura 2b), distinguindo-se de $S$. macrocarpa pelo hábito não ramificado e pelas inflorescências com ramos primários alongados portando unidades terminais racemiformes.

A ocorrência de espécies com área de distribuição restrita relacionadas a uma espécie com distribuição geográfica mais ampla constitui um padrão biogeográfico recorrente (Gentry 1979, Kruckeberg \& Rabinowitz 1985), no entanto não é possível afirmar se as espécies endêmicas constituem casos de neoendemismo, isto é, se são resultado de eventos de especiação recente, ou paleoendemismo, se sua distribuição atual é relictual, resultado da contração de uma distribuição outrora mais ampla (Major 1988). Assim como foi sugerido em Vellozia albiflora Pohl e espécies relacionadas (Mello-Silva 1995), é provável que as espécies endêmicas nos exemplos citados tenham sido originadas independentemente a partir de ancestrais das espécies mais amplamente distribuídas, como é o caso de $S$. gardneri e $S$. macrocarpa.

Além de alguns dos casos supra-citados, Schefflera fruticosa e S. lucumoides também seguem o padrão de distribuição geográfica restrita a apenas uma serra da Cadeia do Espinhaço. A primeira possui distribuição restrita à porção sul da Serra do Cipó (figura 2b), enquanto que a segunda ocorre nos arredores do Pico do Itabirito, no extremo sul da Serra de Espinhaço (figura 2b). Esse padrão de distribuição geográfica é bastante freqüente em espécies típicas de campos rupestres (Giulietti \& Pirani 1988, e consecutivamente corroborado por diversos autores, e.g. Harley 1988, 1995, Mello-Silva 1995, Barros 2004), embora aqui também seja difícil definir se constituem casos de neoendemismo ou paleoendemismo. A origem neoendêmica tem sido mais freqüentemente invocada para explicar os casos de endemismo restrito em campos rupestres da Cadeia do Espinhaço (Harley 1988, Giulietti et al. 2000), no entanto há autores que apontam para a natureza paleoendêmica de algumas dessas espécies, baseando-se no fato das serras onde são encontradas resultam da erosão diferencial do substrato (Arroyo 1976), isto é, foram mais resistentes aos processos de intemperismo e erosão que as regiões inter-serranas atualmente cobertas por cerrados de terras baixas. Dependendo do período de isolamento das serras, entretanto, ambas as hipóteses podem ser válidas, o que torna mais cauteloso avaliar cada caso individualmente.

Hipóteses mais consistentes acerca da origem das espécies endêmicas de campos rupestres surgirão com a obtenção de filogenias robustas de grupos cuja diversificação foi intensa nesses locais. Com a obtenção de dados geográficos precisos, será possível sugerir quais 
teriam sido os principais eventos de vicariância que resultaram em padrões biogeográficos concordantes e qual a importância relativa de eventos de dispersão à longa distância em cada um dos grupos considerados. Também a determinação da idade dos clados nessas filogenias, com base em datação molecular e/ou com eventual incremento dos raros testemunhos fósseis disponíveis, possivelmente permitirá avançar com mais segurança nesse tipo de consideração.

Mata Atlântica - espécies de formações florestais. As espécies florestais de Schefflera do Brasil extra-amazônico possuem áreas de distribuição geográfica bastante variáveis, havendo desde as amplamente distribuídas na Região Neotropical até casos notáveis de endemismo, principalmente em florestas montanas dos Estados do Rio de Janeiro e Espírito Santo. A Mata Atlântica contribui de maneira marcante na diversidade do gênero Schefflera no Brasil (Fiaschi \& Pirani 2005a). Foram registradas neste domínio 13 espécies (10 endêmicas), das quais apenas $S$. aurata não ocorre na região Sudeste do Brasil.

i) Espécies de distribuição ampla. Três das espécies encontradas na Mata Atlântica também habitam formações florestais inseridas em outros domínios, tais como o Cerrado e a Floresta Amazônica (S. calva, S. aff. varisiana e $S$. morototoni), razão pela qual foram aqui denominadas espécies de distribuição ampla. Embora seja endêmica da Mata Atlântica, S. angustissima foi também aqui incluída devido à sua ampla distribuição no bioma.

Schefflera angustissima e $S$. calva são espécies proximamente relacionadas e de difícil distinção entre si (Fiaschi et al. 2007). Schefflera angustissima distribui-se na Planície Litorânea do Brasil e na Serra do Mar, desde a Serra dos Órgãos (Rio de Janeiro), até sua extremidade meridional, no Paraná, estendendo-se pelas matas de restinga até o litoral sul de Santa Catarina (figura $1 b$ ). Há coletas recentes desta espécie em áreas montanhosas mais isoladas, tais como a Serra de Ibitipoca (MG) e as florestas montanas do sul da Bahia. As florestas que revestem as áreas de ocorrência de $S$. angustissima são ombrófilas, caracterizando-se por temperatura e pluviosidade elevadas ao longo de todo o ano (Veloso et al. 1991), e diferindo de maneira marcante das florestas onde vegetam indivíduos de $S$. calva, que em função da sazonalidade climática mais acentuada, são estacionais semideciduais, como é o caso das florestas a oeste da Serra da Mantiqueira. Matas ciliares que atravessam a diagonal Nordeste-Sudoeste de formações secas da América do Sul (Prado \& Gibbs 1993) também contribuem para a expansão de $S$. calva em áreas mais interioranas (figura 1b), corroborando padrão proposto para espécies arbóreas típicas da Mata Atlântica e que invadem o domínio do Cerrado (Oliveira-Filho \& Ratter 1995).

Assim, a distribuição geográfica das duas espécies em questão apresenta certa correlação com as florestas ombrófilas densas e as florestas estacionais e ciliares que ocupavam extensas áreas da região Sudeste do Brasil, embora não haja motivos para considerar esses blocos florestais como floristicamente distintos (Oliveira-Filho \& Fontes 2000, Joly et al. 1999). Além do mais, são bastante raros os locais onde as duas espécies ocorrem em simpatria, como é o caso dos arredores de Curitiba (Paraná) e o Planalto Paulistano (figura 1b). Em todo o caso, as florestas transicionais que revestem este último são resultado da contribuição florística de formações florestais diversas que o teriam ocupado em climas favoráveis pretéritos (Garcia \& Pirani 2001, 2005).

Schefflera aff. varisiana ocorre em florestas montanas da Cadeia do Espinhaço, sul da Bahia, e Espírito Santo. Espécimens coletados no leste do Brasil assemelham-se muito a $S$. varisiana Frodin, encontrada em formações florestais do Vale dos Auaris (Venezuela), na fitocória da "Guayana Venezuelana" (Daly \& Mitchell 2000). Caso correspondam de fato à mesma espécie, esta ilustraria um padrão de distribuição semelhante ao de algumas espécies herbáceas e arbustivas disjuntas entre a Cadeia do Espinhaço e o Planalto das Guianas (Steyermark 1982, Giulietti \& Pirani 1988, Harley 1988). Como evidência adicional, os materiais desta espécie pertencem ao grupo "Crepinella" de Schefflera, cujo centro de diversidade situa-se na porção venezuelana do Planalto das Guianas (Frodin 1995, Fiaschi \& Pirani 2005c, 2007).

$\mathrm{Na}$ Cadeia do Espinhaço Schefflera aff. varisiana ocorre apenas na porção norte da Serra do Cipó (Minas Gerais) e na porção sul da Chapada Diamantina (Bahia) (figura 1e), não havendo até aqui registros de sua ocorrência em locais intermediários como o Planalto de Diamantina e serras do norte da Serra do Espinhaço. Assim, duas hipóteses possíveis para explicar essa disjunção regional seriam: (1) dispersão a longa distância a partir de uma das duas áreas, ou (2) que as populações atuais sejam relictuais de uma distribuição outrora mais ampla ao longo da Cadeia. A segunda hipótese é aqui aceita como a mais provável, devido à baixa evidência de possibilidade de dispersão a longa distância das drupas da espécie. Além disso, hipóteses de disjunções resultantes de alterações geológicas, geográficas ou ecológicas são passíveis de corroboração após a comparação com situações recorrentes em outros taxa, visto que tais mudanças ambientais teriam afetado toda uma biota. Em contraste, as hipóteses de dispersão envolvem estritamente modelos ad hoc individuais para cada espécie e não devem ser adotadas a priori. 
ii) Endemismo - florestas montanas e submontanas. Levando-se em consideração somente as espécies endêmicas das matas costeiras do Domínio da Mata Atlântica, das 10 espécies registradas, sete são referidas apenas para a Região Sudeste do Brasil. Dentre estas, os casos de endemismo mais restrito são exemplificados por Schefflera capixaba, S. grandigemma, S. kollmannii, S. racemifera, S. ruschiana e $S$. succinea, todas encontradas em altitudes superiores a $600 \mathrm{~m}$; a sétima espécie, S. longipetiolata, tem ocorrência em três estados (ES, MG e RJ).

No Estado do Rio de Janeiro merece destaque a ocorrência de $S$. succinea, espécie endêmica de florestas de altitude de Macaé de Cima e arredores, no extremo setentrional da Serra do Mar (figura 1e). Casos de endemismo como esse são freqüentes nessas florestas e sugerem a necessidade de políticas de conservação efetivas para superar o ritmo acelerado com que têm sido derrubadas (Guedes-Bruni \& Lima 1997). Schefflera falcatifoliola (Taub.) Frodin e Schefflera anomala (Taub.) Frodin, consideradas por alguns autores como endêmicas do Rio de Janeiro (Moura 1983, Frodin \& Govaerts 2003), foram aqui consideradas sinônimos de S. angustissima (Fiaschi \& Pirani 2007).

Em função do relevo menos acidentado da porção norte do Estado do Rio de Janeiro e sul do Espírito Santo, situação decorrente da fragmentação da Serra do Mar na região da drenagem do Rio Paraíba e afluentes (Moreira \& Camlier 1977), as matas desse trecho têm sofrido um ritmo bastante acelerado de devastação. No Espírito Santo, a área coberta pela Mata Atlântica encontra-se reduzida a menos de $10 \%$ da cobertura original (Sick \& Teixeira 1979 apud Mori et al. 1981, Pereira 2007). A situação só não é pior porque o conjunto de serras ao sul do Rio Doce, que segundo Fernandes (1995) corresponde a ramificações do sistema da Mantiqueira, ainda preserva fragmentos de mata em áreas particulares, e parques estaduais e federais. Assim como as matas da Serra dos Órgãos (Rio de Janeiro), as matas dessa região, caracterizadas por Ruschi (1950) pela presença freqüente de alguns gêneros [inclusive Schefflera (como Didymopanax)], também devem ser consideradas como um centro de endemismo do gênero Schefflera no domínio da Mata Atlântica, uma vez que abrigam sete espécies, sendo cinco endêmicas: S. capixaba, S. grandigemma, $S$. kollmannii, S. racemifera e S. ruschiana (figuras 1f, 2c, 2d).

Schefflera capixaba, por exemplo, é conhecida de apenas uma coleta na Reserva Biológica de Duas Bocas, em Cariacica (ES), e constitui parte de um grupo que também inclui $S$. longipetiolata e $S$. racemifera (Fiaschi \& Pirani 2007). A primeira distribui-se mais amplamente em florestas ombrófilas da Região Sudeste e ocupa áreas com amplitude altitudinal de até 1000 m (figura 1f), ao passo que a segunda também é conhecida de apenas uma localidade, no Município de Santa Maria de Jetibá, nos arredores de Santa Teresa, Espírito Santo (figura 1f). Evidências morfológicas que sustentam o relacionamento entre essas espécies incluem o hábito monocaule originado pelo modelo de crescimento de Chamberlain (Hallé et al. 1978), e a morfologia estipular. Essas evidências sugerem que este grupo seja proximamente relacionado a $S$. ciliatifolia Fiaschi \& Frodin, e S. plurifolia Fiaschi \& Frodin, duas espécies amazônicas recém descritas (Fiaschi et al. 2008), no entanto não há estudos filogenéticos até o momento que testem adequadamente essa hipótese.

Caso receba confirmação em estudos subseqüentes, o relacionamento aqui proposto tornaria evidente um caso interessante de disjunção entre as florestas ombrófilas Atlântica e Amazônica, fornecendo mais um exemplo da conexão florística entre os dois grandes blocos de florestas ombrófilas do Brasil, já sugerido por autores como Rizzini (1963, 1979), Andrade-Lima (1966, 1982), Prance (1979, 1982), Pirani (1990) e Alves et al. (2003). Demais evidências que suportam esse relacionamento incluem estudos com mamíferos de pequeno porte (Costa 2002), dípteros e primatas (Amorim \& Pires 1996), borboletas (Brown 1987), aves (Cracraft \& Prum 1988), entre outros. Entretanto, vale ressaltar que ao invés de assumir as florestas amazônicas como uma unidade biogeográfica a priori, Amorim (2001) sugeriu tratar-se de uma área biogeográfica composta por dois blocos, apenas um dos quais com conexão histórica com a Mata Atlântica.

Apesar de reconhecida por diversos autores, as hipóteses acerca de como teria se estabelecido essa conexão variam consideravelmente. Enquanto alguns sugeriram que uma rede dendrítica de matas ciliares do Brasil central (ainda hoje existente) teria desempenhado essa função (Oliveira-Filho \& Ratter 1995), outros apontaram para uma ligação mais contínua e extensa pelo nordeste do Brasil, usando como evidência a posição geográfica e composição florística dos brejos de altitude do sertão nordestino (Andrade-Lima 1966, Bigarella et al. 1975). Uma conexão muito mais antiga, via um arco de florestas estendendo-se do norte do Brasil até a África ocidental e a costa atlântica brasileira, antes da abertura final do Oceano Atlântico no Cretáceo, foi proposta por Fernandes \& Bezerra (1990) e Fernandes (2003).

De qualquer modo, as idéias de estabilidade climática moldando a vasta diversidade organísmica na região Neotropical (e.g. Ashton 1969) foram suplantadas por vários modelos invocando altas taxas de especiação relacionadas a fortes contrastes climáticos em períodos 
alternados do Cenozóico (Burnham \& Graham 1999, Haffer 2001). Os recentes avanços nos estudos paleoambientais na América do Sul têm fornecido evidências crescentes de uma dinâmica climática pretérita fragmentando a continuidade da cobertura florestal em partes diversas do continente (e.g. Suguio 1999, Burnham \& Graham 1999, Sant'Anna Neto \& Nery 2005, Oliveira et al. 2005)

iii) Endemismo - Restinga. Além dos elevados níveis de diversidade e endemismo verificados em florestas montanas, o domínio da Mata Atlântica também abriga espécies endêmicas da Planície Costeira, tais como Schefflera aurata e S. selloi, ambas com distribuição restrita às formações vegetais sobre restinga, sendo a primeira conhecida apenas de coletas do norte de Canavieiras até Itacaré, no litoral sul da Bahia, e a segunda comum ao longo da costa do Espírito Santo e do sul da Bahia (figuras 1d, 2c). A distribuição geográfica de ambas está mais ou menos de acordo com uma das três áreas de endemismo de plantas vasculares das florestas ombrófilas da costa atlântica do Brasil, que, segundo Thomas et al. (1998) incluiria as porções ao sul de Salvador (BA) e ao norte do Rio Doce (ES), padrão anteriormente sugerido em espécies de Chrysobalanaceae (Prance 1979). A única exceção trata-se da presença de Schefflera selloi em florestas sobre restinga de Guarapari (ES), local que provavelmente corresponde ao limite sul de distribuição da espécie, dado não haver registros de sua ocorrência no estado do Rio de Janeiro (Araújo 2000).

Considerações finais - As espécies de Schefflera do Brasil extra-amazônico ilustram padrões de distribuição geográfica já referidos para outros organismos. Duas áreas são especialmente ricas em endemismos neste grupo: a Cadeia do Espinhaço em Minas Gerais e as florestas serranas do Espírito Santo e norte do Rio de Janeiro, ambas localizadas nas bordas orientais do Planalto Brasileiro.

Dado que a maioria das espécies do grupo "Didymopanax" são restritas a áreas ao leste da Cordilheira dos Andes, trata-se de um grupo bastante adequado para investigar a biogeografia histórica da região. Hipóteses robustas de relacionamento filogenético entre as espécies do grupo são extremamente necessárias como ponto de partida para avaliar a importância relativa de eventos de vicariância e dispersão responsáveis pelos padrões de distribuição atualmente observados e aqui mapeados.

Agradecimentos - Os autores agradecem à FAPESP pela bolsa de Mestrado concedida ao primeiro autor, ao CNPq pelo apoio com bolsa GDE ao primeiro autor e bolsa de Produtividade em Pesquisa ao segundo, ao programa de doutoramento em "Integrative Life Sciences" da Virginia
Commonwealth University pelo financiamento parcial, aos curadores dos herbários consultados, ao IBAMA e Instituto Florestal de São Paulo, responsáveis pelas unidades de conservação visitadas, e aos revisores e editores do manuscrito.

\section{Referências bibliográficas}

AB'SABER, A.N. 1967. Domínios morfoclimáticos e províncias fitogeográficas no Brasil. Orientação 3:45-48.

ALVES, M.V., THOMAS, W.W. \& WANDERLEY, M.G.L. 2003. Padrões de distribuição geográfica das espécies neotropicais de Hypolytrum Rich. (Cyperaceae). Boletim de Botânica da Universidade de São Paulo 21:265-276.

AMORIM, D.S. 2001. Dos Amazonias. In Introducción a la biogeografía en Latinoamérica: teorías, conceptos, métodos y aplicaciones (J.L. Bousquets \& J.J. Morrone, eds.). UNAM, México, p.245-255.

AMORIM, D.S. \& PIRES, M.R.S. 1996. Neotropical Biogeography and a method for maximum biodiversity estimation. In Biodiversity in Brazil: a first approach (C.E.M. Bicudo \& N.A. Menezes, eds.). CNPq, São Paulo, p.183-219.

ANDRADE-LIMA, D. 1966. Contribuição ao estudo do paralelismo da flora Amazônico-Nordestina. Instituto de Pesquisas Agronômicas de Pernambuco, Boletim Técnico 19:3-30.

ANDRADE-LIMA, D. 1982. Present-day forest refuges in Northeastern Brazil. In Biological diversification in the tropics (G.T. Prance, ed.). Columbia University Press, New York, p.247-251.

ARAUJO, D.S.D. 2000. Análise florística e fitogeográfica das restingas do Estado do Rio de Janeiro. Tese de doutorado, Universidade Federal do Rio de de Janeiro, Rio de Janeiro.

ARROYO, M.T.K. 1976. The systematics of the legume genus Harpalyce (Leguminosae: Lotoideae. Memoirs of the New York Botanical Garden 26:1-80.

ASHTON, P.S. 1969. Speciation among tropical forest trees: some deductions in the light of recent evidence. Biological Journal of the Linnean Society 1:155-196.

BARROS, F. 2004. Distribuição geográfica de orquídeas do Planalto Central do Brasil. In Orquidologia sulamericana: uma compilação científica (F. Barros \& G.B. Kerbauy, eds.). Secretaria do Meio Ambiente, São Paulo, p.147-153.

BIGARELLA, J.J., ANDRADE-LIMA, D. de \& RIEHS, P.J. 1975. Considerações a respeito das mudanças paleoambientais na distribuição de algumas espécies vegetais e animais no Brasil. Anais da Academia Brasileira de Ciências 47:411-464.

BROWN, K.R. 1987. Biogeography and evolution of Neotropical butterflies. In Biogeography and Quaternary history in tropical America (T.C. Whitmore \& G.T. Prance, eds.). Oxford Science Publications, Oxford, p.66-104. 
BURNHAM, R.J. \& GRAHAM, A. 1999. The history of Neotropical vegetation: new developments and status. Annals of the Missouri Botanical Garden 86:546-589.

CASTRO, A.A.J.F., MARTINS, F.R., TAMASHIRO, J.Y. \& SHEPHERD, G.J. 1999. How rich is the flora of Brazilian cerrados? Annals of the Missouri Botanical Garden 86:192-224.

COSTA, L.P. 2002. The historical bridge between the Amazon and the Atlantic Forest of Brazil: a study of molecular phylogeography with small mammals. Journal of Biogeography 30:71-86.

CRACRAFT, J. \& PRUM, R.O. 1988. Patterns and processes of diversification: speciation and historical congruence in some Neotropical birds. Evolution 42:603-620.

CRONQ, Q.C.B. 1998. The ochlospecies concept. In Chorology, taxonomy and ecology of the floras of Africa and Madagascar (C.R. Huxley, J.M. Lock \& D.F. Cutler, eds.). Royal Botanic Gardens, Kew, p.155-170.

DALY, D. \& MICHELL, J. 2000. Lowland vegetation of tropical South America. In Imperfect balance: landscape transformations in the pre-Columbian Americas (D. Lentz, ed.). Columbia University Press. New York, p. 393-453.

FERNANDES, A.G. \& BEZERRA, P. 1990. Estudo fitogeográfico do Brasil. Stylus Comunicações, Fortaleza.

FERNANDES, A.G. 2003. Conexões florísticas do Brasil. Banco do Nordeste, Fortaleza.

FERNANDES, H. de Q.B. 1995. Palmeiras (Palmae) do gênero Bactris nativas do Espírito Santo, Brasil. Dissertação de mestrado, Rio de Janeiro.

FIASCHI, P. 2004. Schefflera aurata, a new species of Araliaceae from southern Bahia, Brazil. Brittonia 56: 357-360.

FIASCHI, P. \& FRODIN, D.G. 2006. A new species of Schefflera (Araliaceae) from Espírito Santo state, Brazil. Kew Bulletin 61:187-191.

FIASCHI, P. \& PIRANI, J.R. 2005a. Four new species of Schefflera J.R. Forst \& G. Forst (Araliaceae) from the state of Espírito Santo, Brazil. Kew Bulletin 60:77-85.

FIASCHI, P. \& PIRANI, J.R. 2005b. Three new species of Schefflera J.R. Forst \& G. Forst (Araliaceae) from the Espinhaço range, Minas Gerais, Brazil. Novon 15:117122.

FIASCHI, P. \& PIRANI, J.R. 2005c. Flora da Serra de Cipó, Minas Gerais: Araliaceae. Boletim de Botânica da Universidade de São Paulo 23:267-275.

FIASCHI, P. \& PIRANI, J.R. 2007. Estudo taxonômico do gênero Schefflera J.R. Forst. \& G. Forst (Araliaceae) na região sudeste do Brasil. Boletim de Botânica da Universidade de São Paulo 25:95-142.

FIASCHI, P., FRODIN, D.G. \& PLUNKETT, G.M. 2008. Four new species of the Didymopanax group of Scheflera (Araliaceae) from the Brazilian Amazon Brittonia 60:274-286.
FIASCHI, P., JUNG-MENDAÇOLLI, S.L., CABRAL, L.P. \& FRODIN, D.G. 2007. Araliaceae. In Flora Fanerogâmica do Estado de São Paulo, vol. 5 (M.G.L. Wanderley, G.J. Shepherd, T.S. Melhem \& A.M. Giulietti, eds.), p.1-16.

FRODIN, D.G. 1995. Neotropical Montane Araliaceae: an overview. In Biodiversity and conservation of Neotropical Montane Forests (S.P. Churchill, H. Baslev, E. Forero \& J.L. Luteyn, eds.). New York Botanical Garden, New York, p.421-430.

FRODIN, D.G. 2004. Araliaceae. In Flowering plants of the Neotropics (N. Smith, S.A. Mori, A. Henderson, D.W. Stevenson \& S.V. Heald, eds.). Princeton University Press, Princeton, p.28-31.

FRODIN, D.G. \& GOVAERTS, R. 2003. World checklist and bibliography: Araliaceae. Royal Botanic Gardens, Kew.

GARCIA, R.J.F. \& PIRANI, J.R. 2001. Estudo florístico dos componentes arbóreo e arbustivo da mata do Parque Santo Dias, São Paulo, SP, Brasil. Boletim de Botânica da Universidade de São Paulo 19:15-42.

GARCIA, R.J.F \& PIRANI, J.R. 2005. Análise florística, ecológica e fitogeográfica do Núcleo Curucutu, Parque Estadual da Serra do Mar (São Paulo, SP), com ênfase nos campos junto à crista da Serra do Mar. Hoehnea 32:1-48.

GENTRY, A.H. 1979. Distribution patterns of Neotropical Bignoniaceae: some phytogeographic implications. In Tropical botany (K. Larsen \& L.B. Holm-Nielsen, eds.). Academic Press, London, p.339-354.

GIULIETTI, A.M. \& PIRANI, J.R. 1988. Patterns of geographic distribution of some plant species from the Espinhaço Range, Minas Gerais and Bahia, Brazil, In Proceedings of a workshop on Neotropical Distribution Patterns (P.E. Vanzolini \& W.R. Heyer, eds.). Academia Brasileira de Ciências, Rio de Janeiro, p.39-69.

GIULIETTI, A.M., HARLEY, R.M., QUEIROZ, L.P. de, WANDERLEY, M.G.L., \& PIRANI, J.R. 2000. Caracterização e endemismos nos campos rupestres da Cadeia do Espinhaço. In Tópicos Atuais em Botânica: Palestras convidadas do 51ํㅡㄹ Congresso Nacional de Botânica (T.B. Cavalcanti \& B.M.T. Walter, orgs.). Embrapa / SBB, Brasília, p.311-318.

GRANVILLE, J.J. 1992. Un cas de distribution particulier: les espèces forestières peri-amazoniennes. Compte-Rendu des Séances de la Societé de Biogéographie 68:1-33.

GUEDES-BRUNI, R.R. \& LIMA, H.C. de. 1997. Mountain ranges of Rio de Janeiro. In Centers of plant diversity. A guide and strategy for their conservation, vol. 3: the Americas (S.D. Davis, V.H. Heywood, O. HerraraMacBryde, J. Villa-Lobos \& A.C. Hamilton, eds.). WWF/IUCN, Cambridge, p.376-380.

HAFFER, J. 2001. Hypotheses to explain the origin of species in Amazonia. In Diversidade biológica e cultural da Amazônia (I.C.B. Vieira, J.M.C. Silva, D.C. Oren \& M.A. D'Incao. orgs.). Museu Paraense Emilio Goeldi, Belém, p.45-118. 
HALlÉ, F., OLDEMAN, R.A.A. \& TOMLINSON, P.B. 1978. Tropical trees and forests. An architectural analysis. Springer-Verlag, New York.

HARLEY, R.M. 1988. Evolution and distribution of Eriope (Labiatae) and its relatives in Brazil. In Proceedings of a workshop on Neotropical distribution patterns (P.E. Vanzolini \& W.R. Heyer, eds.). Academia Brasileira de Ciências, Rio de Janeiro, p.71-120.

HARLEY, R.M. 1995. Introdução. In Flora of the Pico das Almas-Chapada Diamantina, Bahia, Brazil (B.L. Stannard, ed.). Royal Botanic Garden, Kew, p.43-78.

HOLMGREN, P.K., HOLMGREN, N.H. \& BARNETT, L.C. 1990. Index Herbariorum, Part I: The Herbaria of the World. New York Botanical Garden, Bronx, New York.

JOLY, C.A., AIDAR, M.P.M., KLINK, C.A., McGRATH, D.G., MOREIRA, A.G., MOUTINHO, P., NEPSTAD, D.C., OLIVEIRA, A.A., POTT, A., RODAL, M.J.N. \& SAMPAIO, E.V.S.B. 1999. Evolution of the Brazilian phytogeography classification systems: implications for biodiversity conservation. Ciência \& Cultura 51:331348.

KRUCKEBERG, A.R. \& RABINOWITZ. 1985. Biological aspects of endemism in higher plants. Annual Review of Ecology and Systematics 16:447-479.

MAJOR, J. 1988. Endemism: a botanical perspective. In Analytical biogeography: an integrated approach to the study of animal and plant distributions (A.A. Myers \& P.S. Giller, eds.). Chapman \& Hall, London, p.117-146.

MELLO-SILVA, R. 1995. Aspectos taxonômicos, biogeográficos, morfológicos e biológicos das Velloziaceae de Grão Mogol, Minas Gerais, Brasil. Boletim de Botânica da Universidade de São Paulo 14:49-79.

MOREIRA, A.A.N. \& CAMELIER, C. 1977. Relevo. In Geografia do Brasil, IBGE, Rio de Janeiro, v.3, p.1-50.

MORI, S.A., BOOM, B.A. \& PRANCE, G.T. 1981. Distribution patterns and conservation of eastern Brazilian coastal forest tree species. Brittonia 33:233-245.

MOURA, C.A.F. 1983. Estudo taxonômico de espécies brasileiras de Didymopanax Decne. \& Planch. (Araliaceae). Tese de doutorado, Universidade Estadual de Campinas, Campinas.

MYERS, N., MITTERMEIER, R.A., MITTERMEIER, C.G., FONSECA, G.A.B. da \& KENT, J. 2000. Biodiversity hotspots for conservation priorities. Nature 403:853-858.

OLIVEIRA, P.E., BEHLING, H., LEDRU, M.-P., BARBERI, M., BUSH, M., SALGADO-LABORIAU, M.L., GARCIA, M.J., MEDEANIC, S., BARTH, O.M., BARROS, M. A.A. \& SCHEEL-YBERT, R. 2005. Paleovegetação e paleoclimas do Quaternário do Brasil. In Quaternário do Brasil (C.R.G. Souza, K. Suguio, A.M.S.P. Oliveira \& P.E. Oliveira, eds.). Holos Editora, Ribeirão Preto, p.52-74.

OLIVEIRA-FILHO, A.T. \& FONTES, M.A.L. 2000. Patterns of floristic differentiation among atlantic forests in Southeastern Brazil and the influence of climate. Biotropica 32:793-810.
OLIVEIRA-FILHO, A.T. \& RATTER, J.A. 1995. A study of the origin of Central Brazilian forests by the analysis of plant species distribution patterns. Edinburgh Journal of Botany 52:141-194.

OLIVEIRA-FILHO, A.T. \& RATTER, J.A. 2002. Vegetation physiognomies and woody flora of the Cerrado Biome. In The Cerrados of Brazil: Ecology and natural history of a Neotropical savanna (P.S. Oliveira \& R.J. Marquis, eds.). Columbia University Press, New York, p.91-120.

PEREIRA, O.J. 2007. A cobertura vegetal no Estado do Espírito Santo. In Espécies da Flora ameaçadas de extinção no Estado do Espírito Santo (M. Simonelli \& C.N. de Fraga, orgs.). IPEMA, Vitória, p.17-20.

PIRANI, J.R. 1990. Diversidade taxonômica e padrões de distribuição geográfica em Picramnia (Simaroubaceae) no Brasil. Acta Botanica Brasilica 4:19-44.

PLUNKETT, G.M., WEN, J. \& LOWRY II, P.P. 2004. Infrafamilial classifications and characters in Araliaceae: Insights from the phylogenetic analysis of nuclear (ITS) and plastid (trnL-trnF) sequence data. Plant Systematics \& Evolution 245:1-39.

PLUNKETT, G.M., LOWRY II, P.P, FRODIN, D.G. \& WEN, J. 2005. Phylogeny and geography of Schefflera: pervasive polyphyly in the largest genus of Araliaceae. Annals of the Missouri Botanical Garden 92:202-224.

PRADO, D.E. \& GIBBS, P.E. 1993. Patterns of species distributions in the dry seasonal forests of South America. Annals of the Missouri Botanical Garden 80:902-927.

PRANCE, G.T. 1979. The taxonomy and phytogeography of the Chrysobalanaceae of the Atlantic coastal forests of Brazil. Revista Brasileira de Botânica 2:19-39.

PRANCE, G.T. 1982. Forest Refuges: Evidence from woody Angiosperms. In Biological diversification in the tropics (G.T. Prance, ed.). Columbia University Press, New York, p.137-157.

RAPINI, A. 2000. Asclepiadoideae (Apocynaceae). Espinhaço Range, Minas Gerais, Brazil. In Rapid color guide (R.B. Foster, coord.). Environmental \& Conservation Program, Field Museu, Chicago.

RATTER, J.A., BRIDGEWATER, S., ATKINSON, R. \& RIBEIRO, J.F. 1996. Analysis of the floristic composition of the Brazilian Cerrado vegetation II: comparison of the woody vegetation of 98 areas. Edinburgh Journal of Botany 53:153-180.

RIZZINI, C.T. 1963. Nota prévia sobre a divisão fitogeográfica do Brasil. Revista Brasileira de Geografia. 25:1-64

RIZZINI, C.T. 1979. Tratado de Fitogeografia do Brasil, vol. 2: aspectos sociológicos e florísticos. EDUSP, São Paulo.

RUSCHI, A. 1950. Fitogeografia do Estado do Espírito Santo I: considerações gerais sobre a distribuição da flora no Estado do Espírito Santo. Boletim do Museu de Biologia Mello-Leitão. Série Botânica 1:1-353. 
SANT'ANNA NETO, J.L. \& NERY, J.T. 2005. Variabilidade e mudanças climáticas no Brasil e seus impactos regionais. In Quaternário do Brasil (C.R.G. Souza, K. Suguio, A.M.S.P. Oliveira \& P.E. Oliveira, eds.). Holos Editora, Ribeirão Preto, p.28-51.

SIMON, M.F. \& PROENÇA, C. 2000. Phytogeographic patterns of Mimosa (Mimosoideae, Leguminosae) in the Cerrado biome of Brazil: an indicator genus of highaltitude centers of endemism? Biological Conservation 96:279-296.

SOUZA, C.R.G., SUGUIO, K., OLIVEIRA, A.M.S.P \& OLIVEIRA, P.E. (eds.). 2005. Quaternário do Brasil. Holos Editora, Ribeirão Preto.

STEYERMARK, J.A. 1982. Relationships of some Venezuelan forest refuges with lowland tropical floras. In Biological diversification in the tropics (G.T. Prance, ed.). Columbia University Press, New York, p.182-220.

SUGUIO, K. 1999. Geologia do Quaternário e mudanças ambientais. Paulo's Comunicação e Artes Gráficas, São Paulo.
THOMAS, W.W., CARVALHO, A.M.V., AMORIM, A.M. A., GARRISON, J., ARBELÁEZ, A.L. 1998. Plant endemism in two forests in southern Bahia, Brazil. Biodiversity \& Conservation 7:311-322.

TORRES, R.B., MARTINS, F.R. \& GOUVEA, L.S.K. 1997. Climate, soil, and tree flora relationships in forests in the state of São Paulo, southeastern Brazil. Revista Brasileira de Botânica 20:41-49.

VELOSO, H.P., FILHO, A.L.R.R. \& LIMA, J.C.A. 1991. Classificação da vegetação brasileira, adaptada a um sistema universal. IBGE, Departamento de Recursos Naturais e Estudos Ambientais, Rio de Janeiro.

WEN, J., PLUNKETT, G.M., MITCHELL, A.D. \& WAGSTAFF, S.J. 2001. The evolution of Araliaceae: a phylogenetic analysis based on ITS Sequences of Nuclear Ribosomal DNA. Systematic Botany 26:144-167.

WHITE, F. 1962. Geographic variation and speciation in Africa with particular reference to Diospyros. In Taxonomy and Geography (D. Nichols, ed.). The Systematics Association Publication 4, London, p.71-103. 